\title{
Fabric Phase Sorptive Extraction for the Determination of Anthracyclines in Sewage
}

\author{
Sergio Santana-Viera ${ }^{1, *(\mathbb{C})}$, Alejandro Canino-Byreing ${ }^{1}$, María Esther Torres-Padrón ${ }^{1}$, Zoraida Sosa-Ferrera ${ }^{1}{ }^{1}$, \\ José Juan Santana-Rodríguez ${ }^{1}{ }^{\mathbb{D}}$, Abuzar Kabir ${ }^{2,3}{ }^{\mathbb{1}}$ and Kenneth G. Furton ${ }^{2}$ \\ 1 Instituto Universitario de Estudios Ambientales y Recursos Naturales (i-UNAT), Universidad de Las Palmas \\ de Gran Canaria, 35017 Las Palmas de Gran Canaria, Spain; alejandro.canino103@alu.ulpgc.es (A.C.-B.); \\ miriam.torres@ulpgc.es (M.E.T.-P.); zoraida.sosa@ulpgc.es (Z.S.-F.); josejuan.santana@ulpgc.es (J.J.S.-R.) \\ 2 International Forensic Research Institute, Department of Chemistry and Biochemistry, \\ Florida International University, Miami, FL 33199, USA; akabir@fiu.edu (A.K.); kfurton@fiu.edu (K.G.F.) \\ 3 Department of Pharmacy, Faculty of Allied Health Sciences, Daffodil International University, \\ Dhaka 1207, Bangladesh \\ * Correspondence: sergio.viera@ulpgc.es; Tel.: +34-928454425
}

check for updates

Citation: Santana-Viera, S.;

Canino-Byreing, A.; Torres-Padrón, M.E.; Sosa-Ferrera, Z.;

Santana-Rodríguez, J.J.; Kabir, A.; Furton, K.G. Fabric Phase Sorptive Extraction for the Determination of Anthracyclines in Sewage. Separations 2022, 9, 69. https://doi.org/10.3390/ separations 9030069

Academic Editor: Federica Bianchi

Received: 10 February 2022

Accepted: 3 March 2022

Published: 4 March 2022

Publisher's Note: MDPI stays neutral with regard to jurisdictional claims in published maps and institutional affiliations.

Copyright: (C) 2022 by the authors. Licensee MDPI, Basel, Switzerland. This article is an open access article distributed under the terms and conditions of the Creative Commons Attribution (CC BY) license (https:// creativecommons.org/licenses/by/ $4.0 /)$.

\begin{abstract}
Anthracyclines are a group of antineoplastic compounds used to treat acute leukemia and other cancers, and they are excreted after consumption by patients. These chemicals are often found in sewage at very low concentration levels. For this reason, the development of sensitive analytical methodologies capable of determining them at low concentrations is of prime importance. A simple, fast and sensitive analytical method using fabric phase sorptive extraction (FPSE) followed by ultra-high performance liquid chromatography with fluorescence detection (UHPLC-FD) has been developed and validated for the extraction of anthracyclines from sewage samples. FPSE is a green, cheap, simple, selective and rapid sample preparation technique. The different parameters that affect the performance of the FPSE process, including extraction time, eluting solvent, elution time and $\mathrm{pH}$, were optimized. The developed method showed satisfactory reproducibility, with intraday and interday RSD values lower than $15 \%$ for all the compounds and limits of detection between $0.1-0.15 \mu \mathrm{g} \cdot \mathrm{L}^{-1}$. The unique combination of sample preparation by this micro-extraction technique with fluorescence detector have resulted in the satisfactory extraction of highly polar anthracyclines, without any noticeable matrix effect, a very common shortcoming of exhaustive sample preparation technique such as solid phase extraction (SPE) and mass spectrometry.
\end{abstract}

Keywords: antineoplastic compounds; emerging pollutants; fabric phase sorptive extraction; microextraction techniques; ultra-high performance liquid chromatography-fluorescence detector

\section{Introduction}

Since the end of 90s, scientists have warned of the danger that sewage containing antineoplastic compounds can pose adverse effect on human health and well-being and demanded regular monitoring of these chemical entities [1]. These pharmaceutical compounds enter into the sewage mainly after patient's treatment [2-4], and they are known to be carcinogenic, mutagenic, teratogenic and/or fetotoxic $[2,5,6]$. In addition, many authors have demonstrated that several antineoplastic compounds do not degrade in classical wastewater treatment plants (WWTPs) [7-9], not even after passing through the post-treatment regimens [10] and may generate toxic degradation products [1] that finally end up in rivers or seas. Their long-term effects on non-target organisms and their environmental significance are not clear [11], although some researchers have published lowest observed effect concentration in eco-toxicological tests close to the concentrations detected in effluent from WWTPs [12]. For all of the above, they are considered one of the most toxic pharmaceutical compounds used nowadays [13], and must be monitored to ensure a safe ecosystem. 
Anthracyclines are an important group of antineoplastic compounds that act by preventing cell division or disrupting DNA. This group of antineoplastic compounds is mainly used in the therapeutic fight against acute leukaemia, also as a combination therapy for lymphoma and for solid tumours [14].

Different authors have optimised methodologies to determine them at trace levels in sewage by applying solid phase extraction (SPE) procedures that usually requires large sample volumes and long overall sample preparation times. The anthracyclines commonly determined include doxorubicin (DOX), daunorubicin (DAU) and epirubicin (EPI), but other anthracyclines like doxorubicinol (DOXOL) have been also studied.

Recoveries achieved for these compounds using SPE is variable, despite the fact that almost all the authors used the same cartridge (Oasis HLB). Gomez- Canela et al. [15] achieved good relative recoveries: $100 \%$ for DOX, $73 \%$ for DAU and $104 \%$ for EPI. However, most of them, the recovery was ranging from 57-75\% [16-19]. Adapting a previous methodology [15], Franquet-Griell et al. [20] obtained a recovery ranging from $48-75 \%$, but using in river water instead of effluents of WWTPs. In these works, a LC-MS/MS system was used for the determination of the analytes. In another work, Gomez-Canela et al. [21] obtained a recovery of 37\% for EPI using SPE before LC-Orbitrap-MS, attributing the low recovery to the poor extraction performance of the Oasis HLB extraction sorbent. All methodologies, except Rabii et al. [19], used the same cartridge and obtained a range of relative recovery values for these compounds between $37 \%$ to $104 \%$.

Furthermore, SPE is not a very selective technique, and while it preconcentrates the analytes of interest, it also simultaneously preconcentrates the matrix interferents. Microextraction techniques can overcome the drawbacks of SPE extraction. These processes have the advantage of reducing organic solvent usage, lowering sample volume and extraction time, which are beneficial to both, the environment and the researchers. Selective extraction and preconcentration of highly polar analytes like anthracyclines from an overly complex sample matrix such as sewage water pose a great analytical challenge. Among the available extraction and microextraction techniques, fabric phase sorptive extraction (FPSE) is the only sample preparation technique that, by design, combines the exhaustive extraction principle (primary extraction mechanism in SPE) and the equilibrium extraction principle (primary extraction mechanism in SPME). Due to the simultaneous exploitation of two major extraction principles, high primary contact surface area of FPSE membrane and sponge like porous architecture of sol-gel sorbents, FPSE provides high absolute analyte recovery of polar, medium polar and nonpolar analytes with a very fast extraction kinetics. Unlike other sample preparation techniques, the sorbents in FPSE are chemically bonded to the fabric substrate that allows the exposure of the FPSE membranes to any organic solvent to maximize the analyte recovery after the extraction process. FPSE has also eliminated sample pretreatment processes to remove matrix interferents such as filtration and post treatment processes such as solvent evaporation and sample reconstitution from the sample preparation workflow. In order to successfully carry out the extraction and monitoring of antineoplastic compounds, it is important to develop rapid, cheap and sensitive methods that allow their appropriate determination [22].

Most of the authors have used liquid chromatography tandem mass spectrometry (LC-MS/MS) as the separation and determination technique, which allows to measure very low concentrations, but it is expensive and can be strongly influenced by the matrix effect that can produce, in most cases, a significant suppression of the analyte signal. For this reason, it is a parameter that must be evaluated; however, few authors have done so. In this sense, and regarding to anthracyclines, Yin et al. [16] obtained a range of signal suppression in sewage from 6-78\%. On the other hand, Martín et al. [17] had reported practically no matrix effect, ranging from -5 to $20 \%$. Franquet-Griell et al. [20] obtained also a low matrix effect (between -28 and $6 \%$ ) but, in this case, it may be attributed to the studied matrix (river water). Rabii et al. [19] suffered also a significant suppression of the signal of $78 \%$ for EPI using on-line SPE and Gomez-Canela et al. [21] obtained a recovery of $44 \%$ when 
EPI was injected directly into the LC-Orbitrap-MS equipment. They explained the low recovery due to ion suppression or poor water stability, that is, matrix effect.

To the best of our knowledge, only two works have used other extraction techniques different from SPE for anthracyclines determination. Gouveia et al. [23] developed a methodology based on liquid-liquid extraction (LLE) followed by LC-MS for the extraction of 14 antineoplastic compounds including DOX. They obtained a recovery ranging from $105-132 \%$ for DOX in sewage and an instrumental detection limit (IDL) of $6.47 \mathrm{ng} \cdot \mathrm{L}^{-1}$. Souza et al. [24] have developed a microextraction methodology for this family of compounds, based on dispersive liquid-liquid microextraction (DLLME) and HPLC with fluorescence detection. The compounds analysed were Irinotecan, DOX, DAU and EPI. They obtained a recovery range from $74.28-90.01 \%$ in hospital effluents without almost any matrix effect, obtaining a limit of quantification (LOQ) of $1 \mu \mathrm{g} \cdot \mathrm{L}^{-1}$.

In this work, we have optimised and applied FPSE technique [25] to the extraction of three commonly used anthracyclines (DOX, DAU and EPI) prior to their determination by ultra-high performance liquid chromatography with fluorescence detection. A systematic optimisation of the most suitable fabric phase sorptive extraction sorbent chemistry and extraction conditions through a statistical design of experiment (DoE) was carried out.

\section{Materials and Methods}

\subsection{Materials and Reagents}

Sol-gel sorbent-coated FPSE membranes used in the current study were prepared in Florida International University, Miami, Florida, USA. All chemicals, reagents, solvents, organic polymers and sol-gel precursors used in the project were of the highest quality that can be acquired commercially. Methyltrimethoxysilane (MTMOS), trifluoroacetic acid (TFA), acetone, polytetrahydrofuran (PTHF), poly (ethylene glycol) 300, UCON HTF14 and dichloromethane were purchased from Sigma-Aldrich (St. Louis, MO, USA). Cyanopropyl trimethoxysilane, poly(caprolactone-dimethylsiloxane-caprolactone) block polymer and tetramethyl orthosilicate were purchased from Gelest Inc. (Morrisville, PA, USA). Sodium hydroxide, hydrochloric acid and CW20M were purchased from Fisher Scientific (Pittsburg, PA, USA). Deionized water and acetonitrile were obtained from Fisher Scientific (Pittsburg, PA, USA). Muslin cotton fabric (100\% cellulose) was purchased from Jo-Ann Fabric (Miami, FL, USA). An Eppendorf Centrifuge 5415 R (Eppendorf North America Inc., Hauppauge, NY, USA) was used to remove unwanted and interfering micro particles from the sol solutions prior to sol-gel coating on the fabric substrate used to create fabric phase sorptive extraction membrane. A Philips XL30 Scanning Electron Microscope equipped with an EDAX detector was used to obtain SEM images. A Barnstead NANOPure Diamond (Model D11911) deionized water system (Barnstead Inc., Dubuque, IA, USA) was used to obtain deionized water $(18.0 \mathrm{M} \Omega)$.

HPLC-grade methanol (MeOH), MS- grade acetonitrile (ACN), formic acid (F.A.) and ammonia were all obtained from Panreac Quimica (Barcelona, Spain). Ultrapure water used was provided by a Milli-Q system (Milli-pore, Bedford, MA, USA). Cytostatics compounds (DOX, DAU and EPI) were purchased from Cymit-Química (Barcelona, Spain). Stock solutions containing $500 \mathrm{mg} \cdot \mathrm{L}^{-1}$ (DAU and EPI) and $1000 \mathrm{mg} \cdot \mathrm{L}^{-1}$ (DOX) were prepared by dissolving the compound in $\mathrm{MeOH}$. The solutions were stored in glass-stoppered bottles at $-20{ }^{\circ} \mathrm{C}$ in the dark. Working standard solutions were prepared daily. The studied compounds and their properties are shown in Table 1.

Seven different fabric phase sorptive extraction membranes coated with different sorbents were used in this study: sol-gel CW20M, sol-gel PEG300, sol-gel UCON, sol-gel PTHF250, sol-gel Silica, sol-gel CN-CW20M and sol-gel PCAP-PDMS-PCAP. The main features of these sorbents are presented in Table 2 and a photograph of the appearance of the fabrics used is shown in the Figure 1. 
Table 1. Selected compounds and their pertinent physico-chemical properties.

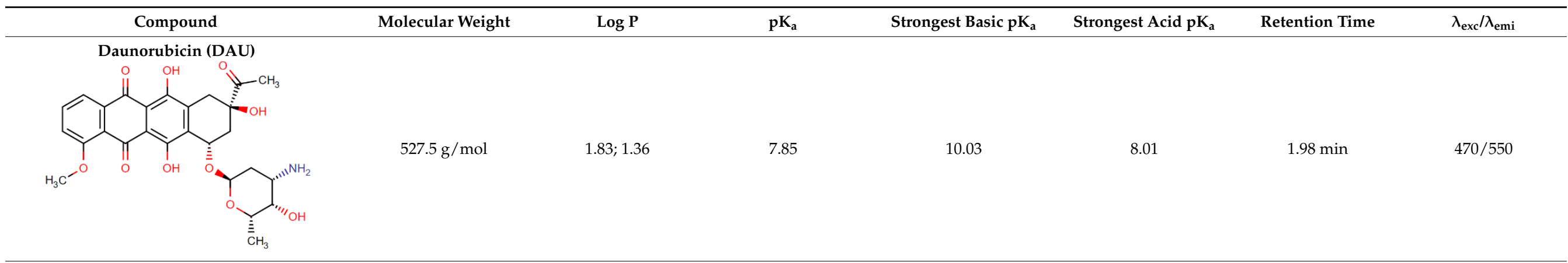

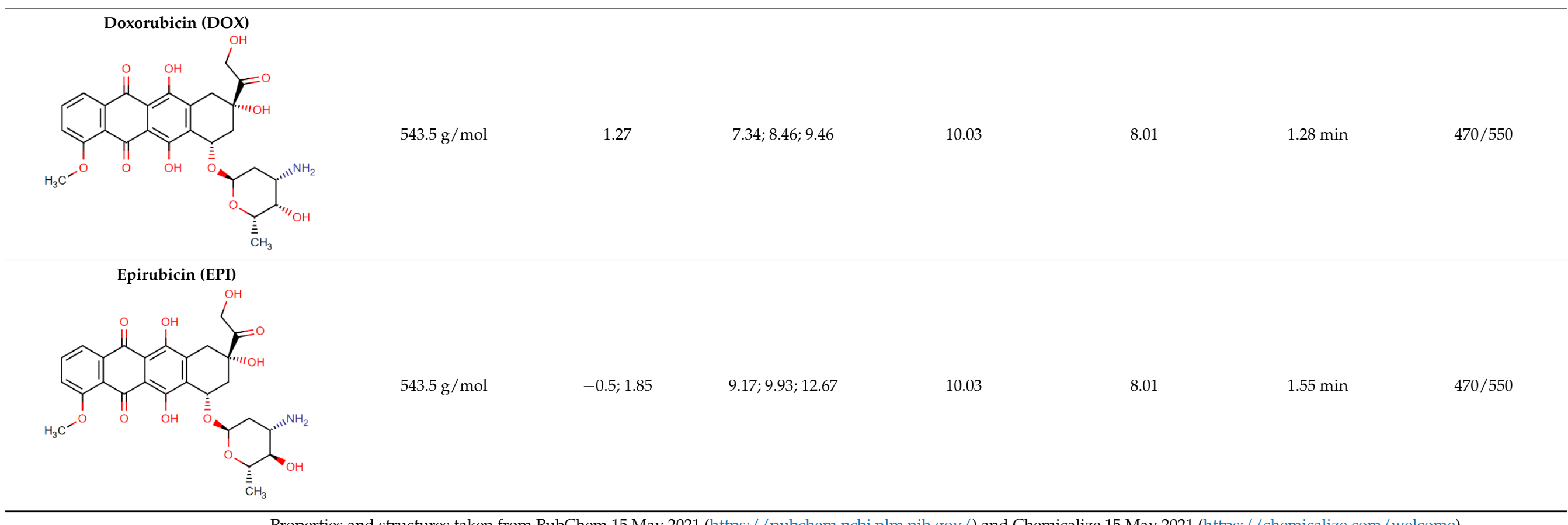

Properties and structures taken from PubChem 15 May 2021 (https://pubchem.ncbi.nlm.nih.gov/) and Chemicalize 15 May 2021 (https://chemicalize.com/welcome). 
Table 2. Selected fabric phase sorptive extraction sorbents used in the current study.

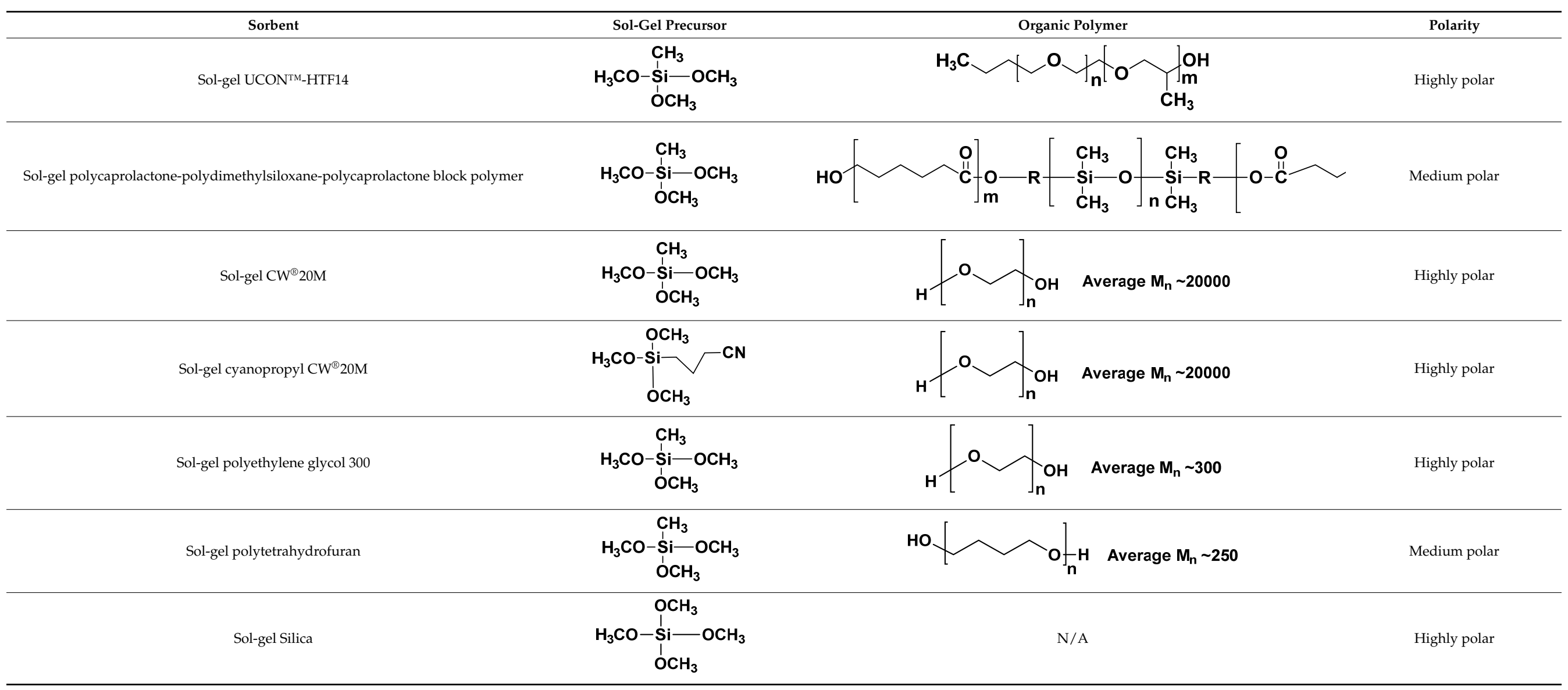




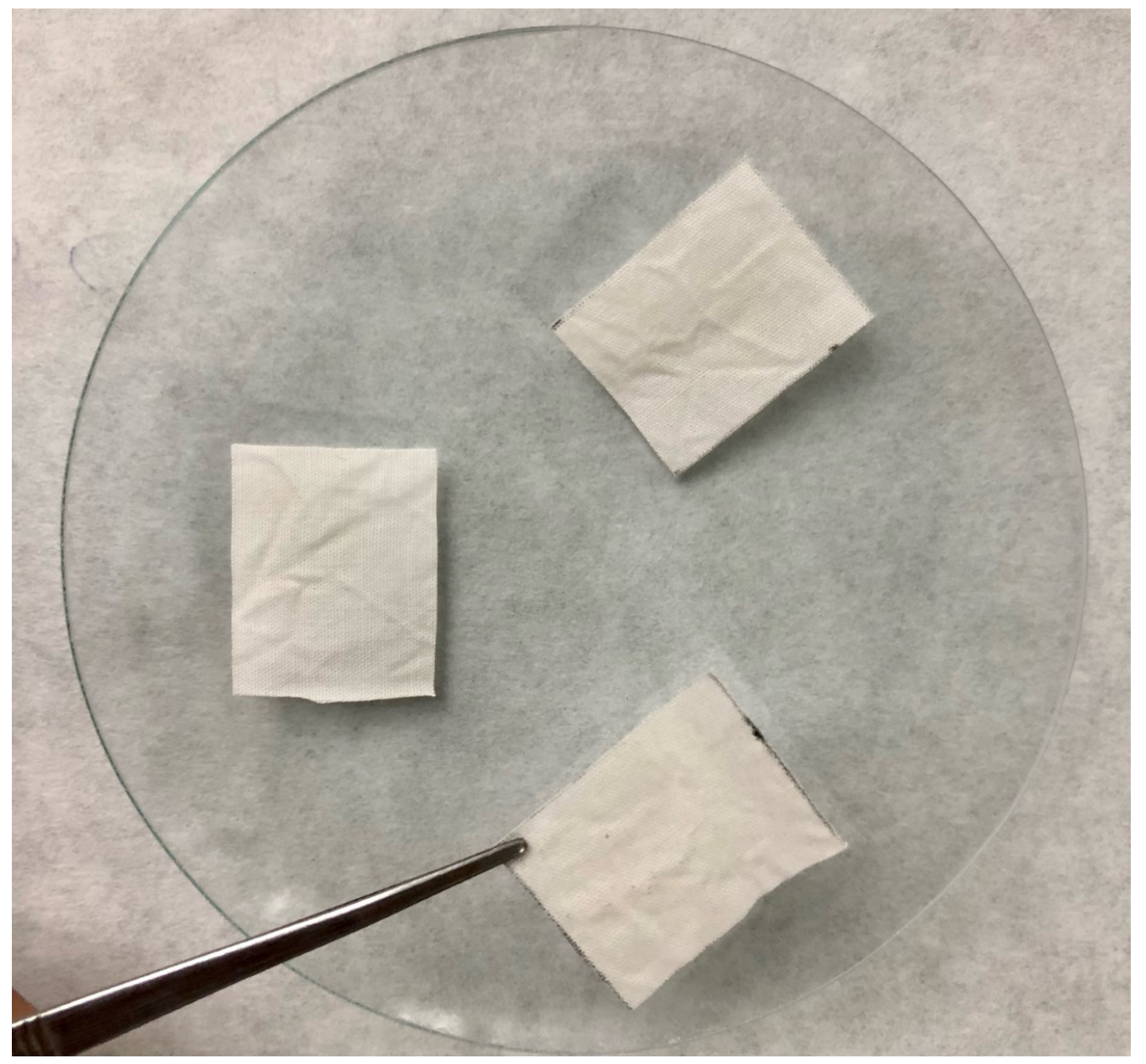

Figure 1. Photograph of the FPSE selected.

\subsection{Creation of Sol-Gel Sorbent Coated Fabric Phase Sorptive Extraction Membrane}

Hydrophilic $100 \%$ cellulose Muslin cotton fabric was used as the substrate for sol-gel sorbent coating. Commercial cotton cellulose fabric contains different surface finishing chemicals as well as other additives to improve the overall appearance of the fabric. However, these chemicals conceal a significant portion of surface hydroxyl functional group and need to be removed to maximize the surface hydroxyl group prior to the sol-gel sorbent coating. The detailed surface treatment process of cellulose fabric is presented elsewhere [26,27].

As the target analytes are highly polar (Table 1), seven different sol-gel sorbents possessing high to moderate polarity were synthesized and evaluated. The sol-gel sorbents include sol-gel CW20M, sol-gel PEG300, sol-gel UCON, sol-gel PTHF250, sol-gel Silica, sol-gel CN-CW20M and sol-gel PCAP-PDMS-PCAP. The formulations of sol-gel CW20M, sol-gel PEG300, sol-gel PTHF250 and sol-gel PCAP-PDMS-PCAP sorbents are described elsewhere [28,29]. Sol-gel Silica sorbent coating was prepared using tetramethyl orthosilicate as the only sol-gel precursor/polymer. Sol-gel UCON and sol-gel CN-CW20M sorbent coatings were prepared using methyl trimethoxysilane and 3-cyanopropyl trimethoxysilane, respectively. The major building blocks of the sol-gel sorbents are presented in Table 2. The detail procedure of sol solution preparation and subsequent coating and post-treatment procedures are presented elsewhere [29] Briefly, the sol solution was prepared by the sequential addition of $5 \mathrm{~g}$ organic/inorganic polymer, $10 \mathrm{~mL}$ acetone: methylene chloride $(1: 1 \mathrm{v} / \mathrm{v}), 5.0 \mathrm{~mL}$ methyltrimethoxysilane and $2.0 \mathrm{~mL}$ trifluoroacetic acid (containing $5 \%$ $v / v$ water). The sol solution was vortexed vigorously after adding each of the ingredients to ensure that the resulting solution becomes homogeneous and particle free. The 
sol solution was then subjected to sonication to remove any trapped air bubbles. Finally, the sol solution was transferred into a $30 \mathrm{~mL}$ amber reaction vessel and a $10 \mathrm{~cm} \times 5 \mathrm{~cm}$ piece of clean and treated cotton fabric was gently immersed into the sol solution. The sol solution was allowed to crate the sol-gel sorbent coating on the fabric substrate for $4 \mathrm{~h}$ at room temperature.

At the end of the sol-gel sorbent coating process, the sorbent coated fabric was removed from the reaction vessel and was stored in a desiccator overnight. Subsequently, the solgel sorbent coated fabric was rinsed with acetone: methylene chloride $(1: 1 v / v)$ under sonication for $30 \mathrm{~min}$. The sol-gel sorbent coated membrane was then air dried for $1 \mathrm{~h}$ and was cut into $1 \mathrm{~cm} \times 1 \mathrm{~cm}$ pieces. The FPSE membranes were then stored in an air-tight container until their application in sample preparation.

\subsection{Instrumentation and Chromatographic Conditions}

Used instrument include an ultra-high performance liquid chromatography system equipped with an ACQUITY Quaternary Solvent Manager (QSM), an autosampler, a column manager and a fluorescence detector (UHPLC-FD) from Waters (Barcelona, Spain). The excitation and emission wavelengths selected were 470 and $550 \mathrm{~nm}$ for all compounds.

The analytical column was a $50 \mathrm{~mm} \times 2.1 \mathrm{~mm}$, Phenomenex Luna Omega column with a particle size of $1.7 \mu \mathrm{m}$ (Phenomenex, Madrid, Spain) operating at room temperature. The sample volume injected was $10 \mu \mathrm{L}$, and the analyte separation was carried out using water $(\mathrm{A})$ and $\mathrm{ACN}(\mathrm{B})$ both with $0.1 \%(v / v)$ of formic acid at a flow rate of $0.4 \mathrm{~mL} \cdot \mathrm{min}^{-1}$ in gradient mode. The gradient started at a 70:30 $(v / v)$ mixture of $\mathrm{A}$ : B and remained in this composition for $0.3 \mathrm{~min}$. Then, it was changed to $15: 85(v / v)$ in $0.7 \mathrm{~min}$ and remained in this composition from 1 to $1.4 \mathrm{~min}$. After that, the percentage of A was decreased again to $0: 100(v / v)$ in $0.1 \mathrm{~min}$ and remained in this composition for $0.5 \mathrm{~min}$. Mobile phase A was increased from $2.0 \mathrm{~min}$ to $3.5 \mathrm{~min}$ to achieve the initial composition 70:30 (v/v) A:B and remained in this composition for $1.5 \mathrm{~min}$ to equilibrate the pressure, achieving the chromatographic separation in $5 \mathrm{~min}$.

\subsection{Fabric Phase Sorptive Extraction Procedure}

Before analysis, FPSE membranes were preconditioned in $2 \mathrm{~mL}$ of a mixture $\mathrm{MeOH} / \mathrm{ACN}$ $(50: 50, v / v)$ for $5 \mathrm{~min}$ and then in $2 \mathrm{~mL}$ of Milli-Q water for another $5 \mathrm{~min}$. After that, the FPSE membrane was placed in contact with the $20 \mathrm{~mL}$ of the sample (adjusted at $\mathrm{pH}=3$ ) for $15 \mathrm{~min}$ and stirred at $1000 \mathrm{rpm}$. After extraction, analytes were eluted with $1 \mathrm{~mL}$ of $\mathrm{MeOH} / \mathrm{ACN}(50: 50, v / v)+10 \%$ F.A. for $4 \mathrm{~min}$. The elution process was repeated and then the solvent was collected together, dried with a gentle stream of nitrogen and reconstituted in $1 \mathrm{~mL}$ of $\mathrm{MeOH} / \mathrm{ACN}(50: 50, v / v)$ for the analysis. All analyses were done in triplicate.

\subsection{Sample Collection}

Sewage samples were collected from the sewage treatment plant of Las Palmas de Gran Canaria (Canary Islands, Spain). The samples were acidified to a pH between 2.5-3 and stored at $4{ }^{\circ} \mathrm{C}$ until analysis. Before analysis, samples were filtered using $0.45 \mu \mathrm{m}$ filter.

\section{Results and Discussion}

\subsection{Chemistry and Selectivity of Sol-Gel Poly (caprolactone-dimethylsiloxane-caprolactone) Coated} FPSE Membrane

Commercially available sample preparation techniques, including solid phase microextraction (SPME), stir bar sorptive extraction (SBSE) and microextraction by packed sorbent (MEPS) often utilize pristine organic or inorganic polymers such as polydimethylsiloxane, poly(ethylene glycol), octadecylsilane as the sorbents. These pristine polymers possess their own intrinsic selectivity/affinity towards the target analytes and cannot be modified/improved to enhance the extraction efficiency. FPSE addresses the selectivity stalemate by uniquely incorporating three selectivity determinants in the device design: (a) a hydrophobic/hydrophilic fabric substrate; (b) an organic or inorganic polymer; and 
(c) a sol-gel precursor to chemically bind the polymer to the fabric substrate. The overall selectivity of the FPSE membrane depends on the nature of these three building blocks and can be easily modified or improved based on the analytical need. For example, application of tetramethyl orthosilicate in sol-gel silica coating transformed the coating into highly polar. Application of methyl trimethoxysilane in most of the sol-gel sorbents provided additional interaction via London dispersion. The utilization of 3-cyanopropyl trimethoxysilane in sol-gel CN-CW20M was to make the resulting sorbent more polar. All sol-gel sorbents were prepared using cotton cellulose fabric as the substrate. The hydrophilic nature of cotton cellulose fabric and its possession of abundant surface hydroxyl groups to anchor the sol-gel sorbent network with the substrate justify its selection as the substrate from a large number of substrate candidates.

Unlike commercial sample preparation techniques, the analytes extraction/preconcentration in FPSE is primarily carried out by different intermolecular interactions such as dipole-dipole interactions, London dispersion and hydrogen bonding between the analytes and the sol-gel based functional sorbents. Sol-gel derived sorbents are inherently porous with sponge-like surface morphology. As such, aqueous solution carrying the target analytes can easily permeate though the spongy sol-gel sorbent, interact rapidly via different intermolecular interactions and consequently get extracted. The built-in-porosity of the fabric substrate is retained even after the sol-gel sorbent coating. As a result, the aqueous sample can easily pass though the FPSE device that mimics a solid phase extraction disk. The through pores of the FPSE membrane also significantly contribute to accelerate the extraction kinetics. As can be seen in Table 1, all three analytes are highly polar. It is logical to expect that polar sorbent such as sol-gel CN-CW20M, sol-gel CW20M, sol-gel PEG 300 would offer higher extraction efficiency compared to medium polar sorbent sol-gel poly (CAP-DMS-CAP). However, as the results demonstrate (Table S1), sol-gel poly(CAP-DMS-CAP) was found as the optimum sorbent when both the sample volume and extraction time were taken into consideration. A closer look into the molecular make up of both the analytes (Table 1) and the polymers (Table 2) reveal that the analytes and poly(CAP-DMS-CAP) possess a large number of hydrogen donors and acceptors. In addition to other intermolecular interactions, hydrogen bonding interactions between the analytes and the FPSE membrane may have played an important role in the extraction mechanism, resulting in the high extraction efficiency of highly polar analytes on a medium polar sol-gel sorbent. The presented results again validate the necessity of sol-gel derived advanced sorbent systems possessing numerous and diversified functional groups.

\subsection{Optimization of Fabric Phase Sorptive Extraction}

The efficiency of sorption primarily depends upon the characteristics of the sorbent material used as well as the physicochemical properties of the analytes' dissolution and the experimental conditions. Thus, the adsorption study was made using the seven fabric displays and an experimental design $2^{3}$ with the variables that the most affect the adsorption. The variables and levels chosen were extraction time (15 and $30 \mathrm{~min}$ ), sample volume (10 and $20 \mathrm{~mL}$ ) and $\mathrm{pH}$ (3 and 9). The adsorption efficiency was calculated by the difference in the concentration of the spiked water before and after extraction. An aliquot of the sample is taken before the extraction and analyzed. After, the fabric is immersed in the sample during the extraction time and another aliquot of the water sample is taken for analysis. Results of all the fabrics tested are shown in the Supplementary Material (Table S1).

As can be seen in the obtained results, the adsorption procedure in FPSE is strongly conditioned by the selected variables. The variation of one of the variables, although the other two remain constant, can lead to a considerable reduction in the adsorption capacity of the fabric. This condition makes it difficult to draw conclusions and predict the behavior.

In general, the best adsorption results were obtained at $\mathrm{pH}=3$, since in these conditions the analytes are in neutral form. However, for the fabrics CW20M and PEG 300 the better results were obtained at $\mathrm{pH}=9$. On the other hand, the fabric PTHF 250 was not greatly 
affected by this variable, since good adsorption results were obtained when the extraction time and the volume of sample were low, regardless of $\mathrm{pH}$. For most of cases, an extraction time of 30 min obtained better results, but some fabrics, such as SICA and PCAP-DMS-CAP, obtained the best results with an extraction time of $15 \mathrm{~min}$. Finally, in relation to the sample volume, most of the fabrics obtained a better adsorption efficiency when $10 \mathrm{~mL}$ was used. However, some fabrics, such as UCON and PCAP-DMS-CAP, achieved their best results when $20 \mathrm{~mL}$ was used.

Good adsorption efficiencies ( $>90 \%$ ) were achieved in all fabrics tested under at least one of the conditions. Hence, for the selection of the fabric in the following tests, different factors were considered. A bigger sample volume would imply a higher preconcentration factor, so a fabric that obtained good results with $20 \mathrm{~mL}$ as sample volume was sought. On the other hand, a shorter extraction time implies a faster methodology, so a fabric that obtained the best results when extracted for 15 min was selected. With these considerations in mind, the fabric PCAP-DMS-CAP was selected for the next stage. Therefore, extraction conditions were set as follow: extraction time $=15 \mathrm{~min}$., sample volume $=20 \mathrm{~mL}$ and $\mathrm{pH}=3$. In these conditions a mean adsorption $(n=8)$ of $80 \pm 9 \%$ of DOX, $85 \pm 7 \%$ of DAU and $87 \pm 7 \%$ of EPI on the selected fabric was obtained. All these results are presented in Figure 2 .

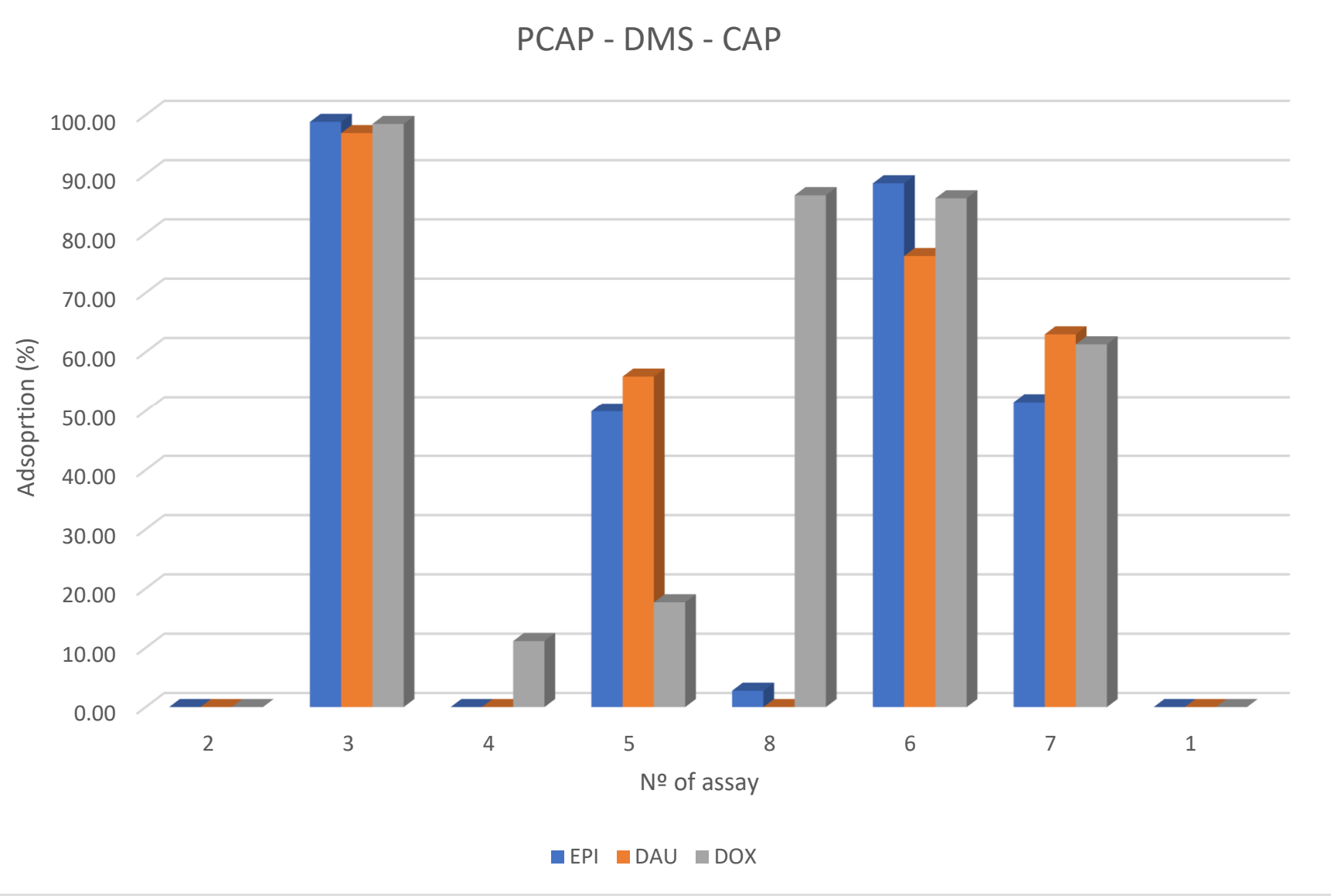

Figure 2. Results of the first experimental design in the selected fabric (PCAP-PDMS-PCAP).

\subsection{Optimization of Desorption Process}

After adsorption conditions are defined, it is necessary to optimize the desorption conditions. To perform that, a $2^{3}$ experimental design with the variables that most affect the elution process was done. The variables selected in this step were the elution time ( 3 and $7 \mathrm{~min}$ ), the elution volume $(0.5$ and $1.5 \mathrm{~mL}$ ) and the solvent type $(\mathrm{MeOH}$ and $\mathrm{ACN})$. Recovery was measured by comparing the signal of a standard with the signal of the eluted. 
After completing the whole design, a statistical study of the results was carried out through Pearson's correlation and the $p$-value. It was found that all the variables affect the process, as indicated by the $p$-value; however, Pearson's correlation did not indicate which value should take the variable (Table 3). Regarding to Pareto charts, it was only found for DOX that the pairwise combination of the variables was significant. EPI and DAU present a similar pattern, but their graphs were below the line of significance. For that reason, it was decided to select the intermediate values of each variable. In this sense, a new experimental design with $3^{2}$ was prepared. The variables selected were solvent $(\mathrm{MeOH}$, $\mathrm{ACN}$ and $\mathrm{MeOH} / \mathrm{ACN}$ (50:50)) and elution time (4, 7 and $10 \mathrm{~min}$.). A value of $1 \mathrm{~mL}$ was set for the elution volume due to that volume of $0.5 \mathrm{~mL}$ did not completely cover the fabric, which can lead to recovery and reproducibility errors. Results of this second experimental design are shown in Figure 3. In the Figure, the solvents are codified as follows: $\mathrm{MeOH}=1$, $\mathrm{MeOH} / \mathrm{ACN}(50: 50)=2$ and $\mathrm{ACN}=3$.

Table 3. Statistical results of the desorption tests.

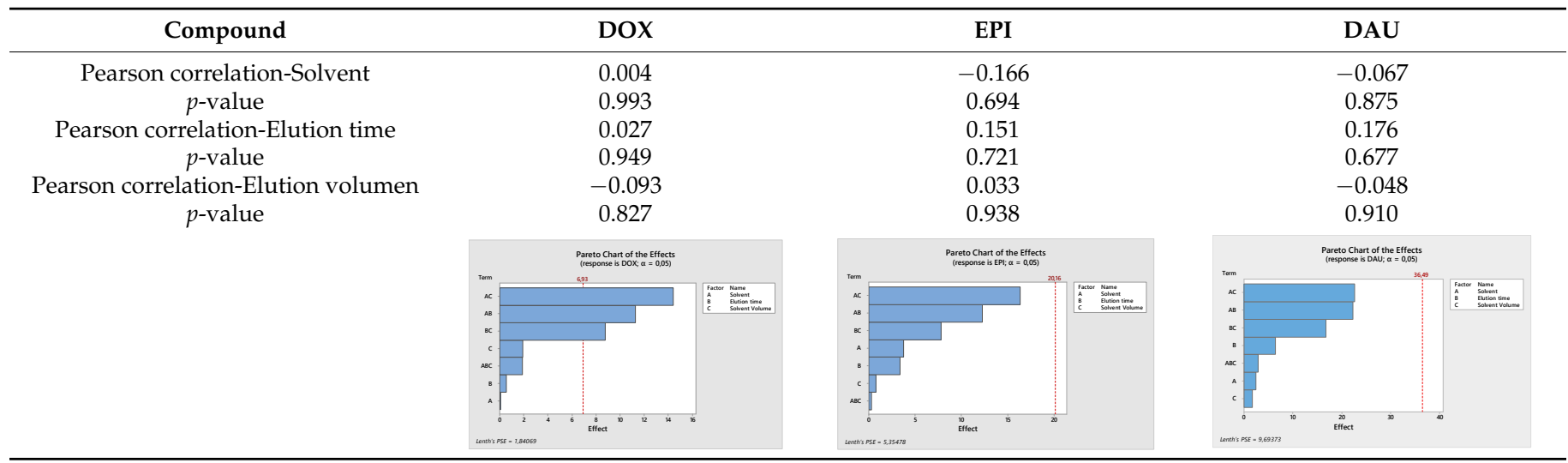

As shown, the mixture of $\mathrm{MeOH} / \mathrm{ACN}$ (50:50) achieved the best recovery values; however, the desorption of the analytes from the fabric is still not complete. FPSE is mainly an equilibrium extraction process, and the results show that, depending on the desorption time, the recovery of the analytes varies, possibly due to adsorption/desorption phenomena that take place until equilibrium is reached. More in depth, it was found that low desorption times benefit the elution, as can be seen in Figure 2, for all of the compounds. Taking into account the results achieved so far, it was decided to set the desorption solvent as a mixture of 50:50 of $\mathrm{MeOH} / \mathrm{ACN}$, and to test lower desorption times. Thus, the elution time was set to $4 \mathrm{~min}$. Minor times did not achieve better recoveries, probably because to reach equilibrium, more time is needed.

In order to increase the desorption efficiency, a new strategy was adopted to increase the solubility of the analytes in the solvent. The $\mathrm{pH}$ of the eluent was modified with ammonia or F.A. At first, 5\% of ammonia and F.A. was tested, finding that a reduction in $\mathrm{pH}$ due to the addition of F.A. favored the recoveries and the increasing of the $\mathrm{pH}$ due to the ammonia did not improve the recovery; so the percentage of F.A. was increased until reaching the inflection point. The maximum desorption efficiency was found when $10 \%$ of F.A. was used. Then, with the selected eluent, a desorption in several steps was tried for even better elution. In it, the fabric was eluted with $1 \mathrm{~mL}$ of $\mathrm{MeOH} / \mathrm{ACN}$ (50:50) + 10\% F.A. for $4 \mathrm{~min}$ several times. It was found that the best desorption efficiency was achieved after carried out 2 elution steps. Then, the elutes were collected together, dried with nitrogen and reconstituted in $1 \mathrm{~mL}$ of $\mathrm{MeOH} / \mathrm{ACN}$ (50:50) before injection. 

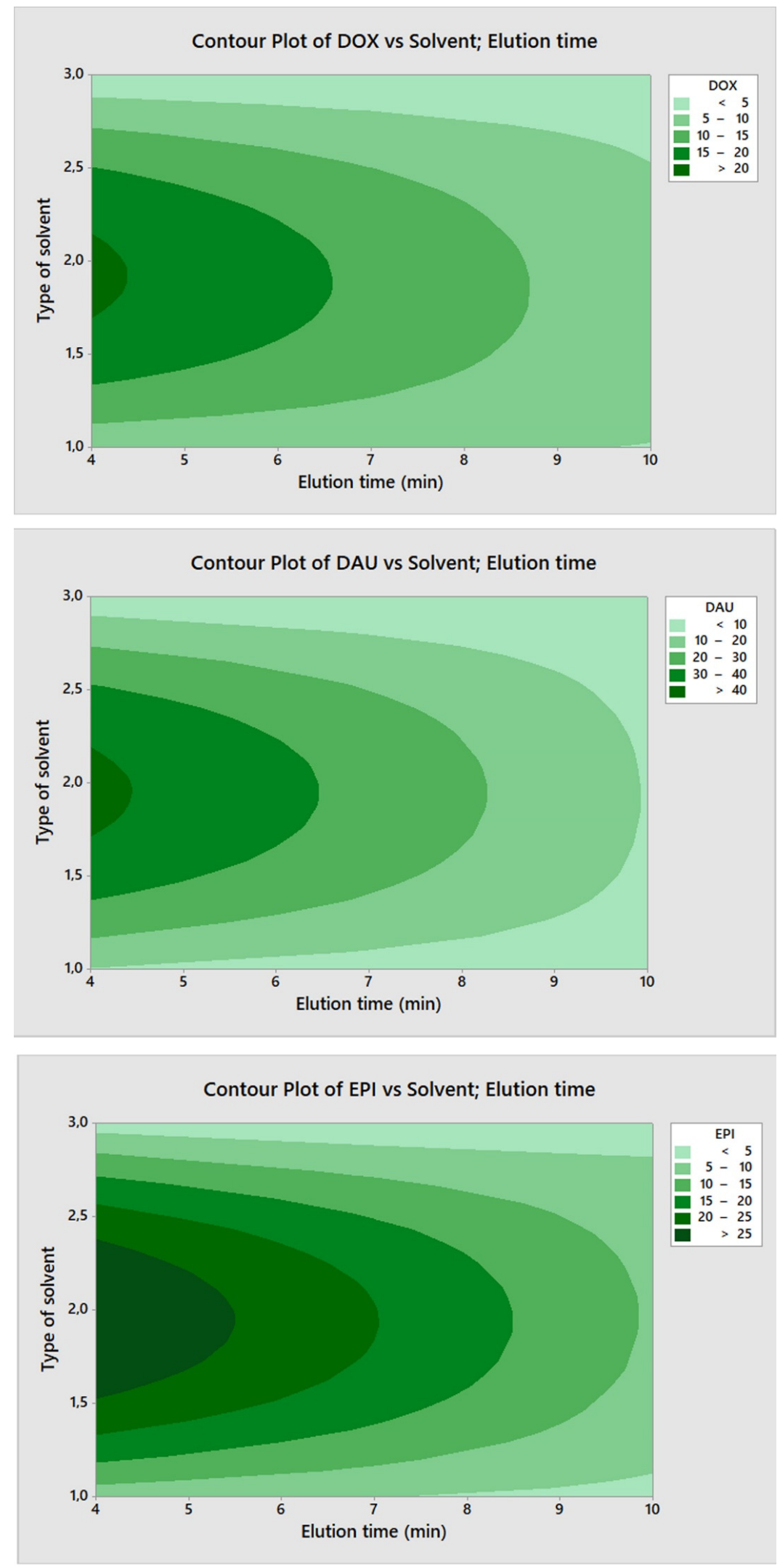

Figure 3. Contour plots of the 32 experimental design in the desorption tests. Solvents are codified as follows: $\mathrm{MeOH}=1, \mathrm{MeOH} / \mathrm{ACN}(50: 50)=2$ and $\mathrm{ACN}=3$. 
In summary, the optimized procedure consists in immersing the selected fabric (PCAPDMS-CAP) in $20 \mathrm{~mL}$ of the sample at $\mathrm{pH}=3$ for $15 \mathrm{~min}$ and stir at $1000 \mathrm{rpm}$. After that, the fabric is immersed in $1 \mathrm{~mL}$ of $\mathrm{MeOH} / \mathrm{ACN}(50: 50)+10 \%$ F.A. for $4 \mathrm{~min}$, twice. The eluted are collected together, dried with nitrogen and reconstituted in $1 \mathrm{~mL}$ of $\mathrm{MeOH} / \mathrm{ACN}$ (50:50, $v / v)$. Taking into account that the procedure started with $20 \mathrm{~mL}$ of the sample and it was preconcentrated up to $1 \mathrm{~mL}$, a preconcentration factor of 20 was reached. This preconcentration factor was taken into account for the calculation of the analytical parameters.

\subsection{Analytical Figures of Merit}

To corroborate the linearity of the method, an external calibration curve was performed. In it, the relationship of peak area and the concentration of each compound was stablished. A linearity with a correlation coefficient (r2) higher than 0.990 was obtained for a concentration range from 25 to $500 \mu \mathrm{g} \cdot \mathrm{L}^{-1}$ for all compounds.

To study the reproducibility and repeatability of the whole method FPSE-UHPLC-FD, intraday and interday studies were done at two level of concentrations $\left(10 \mu \mathrm{g} \cdot \mathrm{L}^{-1}\right.$ and $25 \mu \mathrm{g} \cdot \mathrm{L}^{-1}$, taking into account the preconcentration factor). The intraday relative standard deviation (RSD) was calculated by extracting 6 samples in the same day $(n=6)$, while the interday RSD was calculated by extracting 3 samples $(n=3)$ in 3 different days. Relative recovery was calculated taking into account the mean adsorption of the compounds into the fabric and in the same levels as before. It was calculated as the ratio between the extract of a spiked sample and a MeOH/ACN (50:50) standard. Limit of Detection (LOD) and Limit of Quantification (LOQ) were calculated as the signal to noise ratio higher than 3 and 10, respectively. Table 4 show the analytical figures of merit obtained from the composite method.

Table 4. Analytical figures of merit.

\begin{tabular}{ccccc}
\hline & & EPI & DAU & DOX \\
\hline Relative recovery (Milli-Q water), (\%) & $10 \mu \mathrm{g} \cdot \mathrm{L}^{-1}$ & 51 & 54 & 60 \\
& $25 \mu \mathrm{g} \cdot \mathrm{L}^{-1}$ & 39 & 45 & 58 \\
RSD Intraday (Milli-Q water), (\%) & $10 \mu \mathrm{g} \cdot \mathrm{L}^{-1}$ & 15 & 10 & 10 \\
& $25 \mu \mathrm{g} \cdot \mathrm{L}^{-1}$ & 6 & 7 & 9 \\
RSD Interday (Milli-Q water), (\%) & $10 \mu \mathrm{g} \cdot \mathrm{L}^{-1}$ & 11 & 10 & 11 \\
& $25 \mu \mathrm{g} \cdot \mathrm{L}^{-1}$ & 12 & 6 & 9 \\
LOD & $\mu \mathrm{g} \cdot \mathrm{L}^{-1}$ & 0.12 & 0.10 & 0.15 \\
LOQ & $\mu \mathrm{g} \cdot \mathrm{L}^{-1}$ & 0.41 & 0.33 & 0.49 \\
\hline
\end{tabular}

It was found that the relative recoveries in Milli-Q water vary between 39 and 60\%, depending on the compound, since it is an equilibrium process. Regarding the intraday and interday RSD, satisfactory values were found for all compounds at both levels. RSD values were lower than $15 \%$ for all the compounds in the two levels tested. Finally, LODs and LOQs suitable for the purpose of this work were achieved, ranging from $0.10-0.15 \mu \mathrm{g} \cdot \mathrm{L}^{-1}$ and $0.33-0.49 \mu \mathrm{g} \cdot \mathrm{L}^{-1}$, respectively. Comparing these results with those obtained before using microextraction techniques and FD, we have achieved a LOQ 2-3 times lower.

\subsection{Application to Sewage Samples}

To apply the developed methodology, sewage samples were analysed. However, the target compoundsof this study were not detected in any of the analyzed samples.

Therefore, in order to prove the applicability of the method, sewage samples were spiked at the same levels as the analytical parameters were done. It was found that the variation of the signal, depending on the compound and the concentration, varied from $80-110 \%$, as can be seen in Table 5 and Figure 4. Unlike SPE extractions, which in many cases suffer significant signal suppression, the values obtained with this procedure indicate a great selectivity of the technique, as very low variations due to the matrix effect were obtained. 
Table 5. Relative recovery data on spiked wastewater samples.

\begin{tabular}{ccccc}
\hline & & EPI & DAU & DOX \\
\hline \multirow{2}{*}{$\begin{array}{c}\text { Relative } \\
\text { recovery (\%) }\end{array}$} & $10 \mu \mathrm{g} \cdot \mathrm{L}^{-1}$ & 104.7 & 108.5 & 84.5 \\
\cline { 2 - 5 } & $25 \mu \mathrm{g} \cdot \mathrm{L}^{-1}$ & 85.2 & 83.8 & 79.8 \\
\hline
\end{tabular}

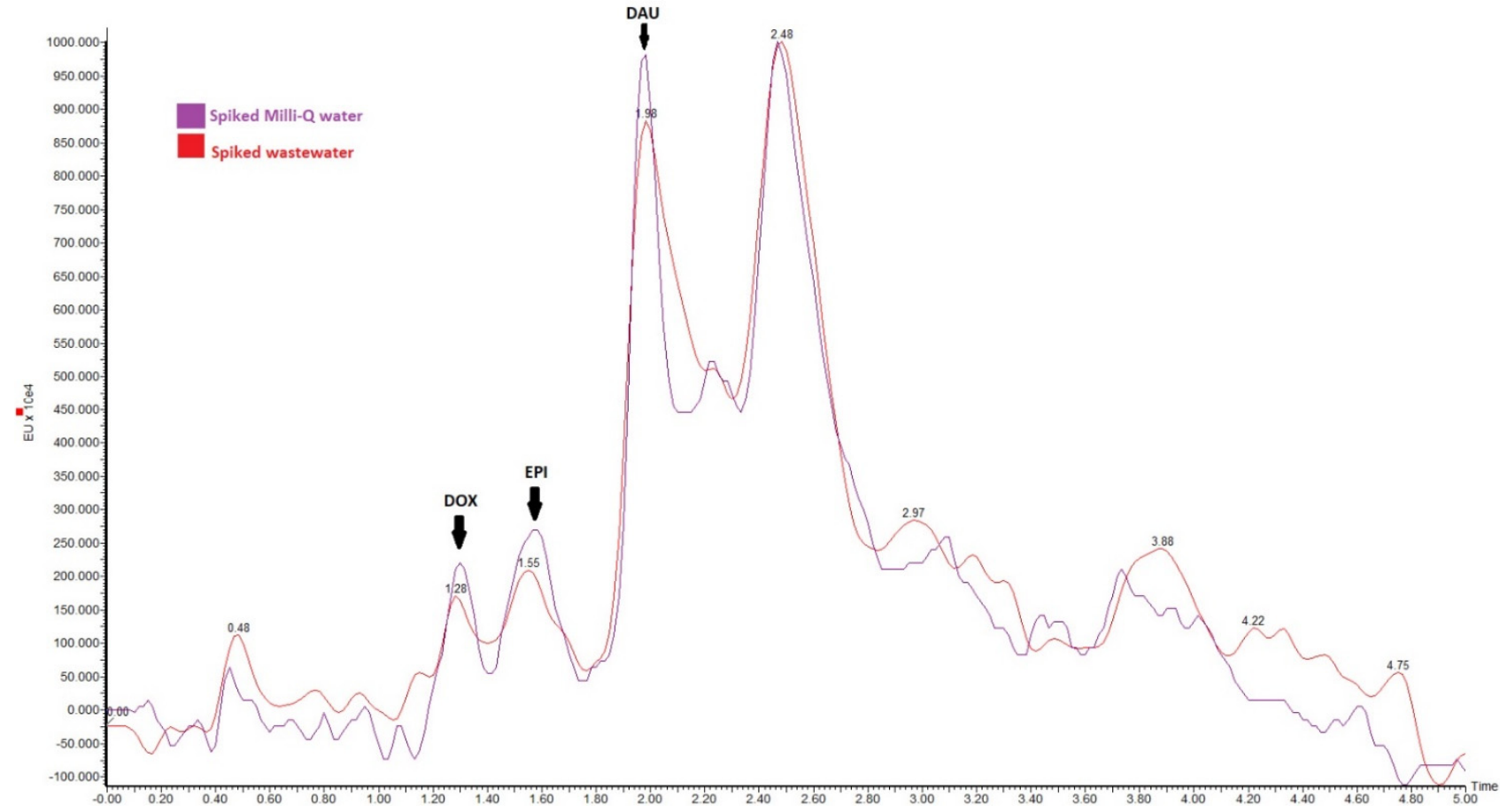

Figure 4. Chromatograms of spiked Milli-Q water vs. spiked wastewater at the lowest point of the analytical parameters.

\section{Conclusions}

The available variety of sorbents for FPSE allows the selection of the most suitable for the mixture of analytes. Therefore, the optimization of a FPSE methodology leads to a low matrix effect, fast and easy extraction and low cost procedure.

This extraction technique was applied to the extraction and preconcentration of anthracyclines, a group of antineoplastic compounds used as chemotherapy treatment against different types, from sewage, prior to their determination by UHPLC-FD. In the optimal conditions, the extraction procedure obtains low RSD deviation, both intraday and interday, lower than $15 \%$ for all the compounds. Due to it being mainly an equilibrium process, mean recoveries ranging from $39 \%$ to $60 \%$ in Milli-Q water were obtained. Nevertheless, good LODs and LOQs were achieved, between $0.1-0.15 \mu \mathrm{g} \cdot \mathrm{L}^{-1}$ and $0.33-0.49 \mu \mathrm{g} \cdot \mathrm{L}^{-1}$, respectively. As a final point, this methodology was applied to sewage samples; however, analytes were not detected in the samples analyzed. Nevertheless, spiked samples prove the applicability of this fast method to a group of pharmaceuticals poorly studied, obtaining recoveries close to those obtained in Milli-Q water and without matrix effect, a common drawback of SPE and LC-MS/MS.

Supplementary Materials: The following supporting information can be downloaded at: https:/ / www.mdpi.com/article/10.3390/separations9030069/s1, Table S1: Adsorption results (efficiency, \%) of the selected compounds on all FPSE membranes studied. 
Author Contributions: S.S.-V.: conceptualization and investigation, laboratory experiments and validation, analysis of results, application of statistical techniques, writing-original draft. A.C.-B.: investigation, laboratory experiments. M.E.T.-P.: conceptualization, administration, analysis of results, writing and reviewing. Z.S.-F.: conceptualization, administration, analysis of results, writing and reviewing. J.J.S.-R.: conceptualization, administration, analysis of results, writing and reviewing. A.K.: investigation, laboratory experiments, writing and reviewing. K.G.F.: investigation, writing and reviewing. All authors have read and agreed to the published version of the manuscript.

Funding: This research received no external funding.

Acknowledgments: Sergio Santana-Viera would like to thank the University of Las Palmas de Gran Canaria (Spain) for his grant.

Conflicts of Interest: The authors declare no conflict of interest.

\section{References}

1. Kiffmeyer, T.; Götze, H.-J.; Jursch, M.; Lüders, U. Trace Enrichment, Chromatographic Separation and Biodegradation of Cytostatic Compounds in Surface Water. Fresenius J. Anal. Chem. 1998, 361, 185-191. [CrossRef]

2. Kümmerer, K.; Al-Ahmad, A.; Bertram, B.; Wießler, M. Biodegradability of Antineoplastic Compounds in Screening Tests: Influence of Glucosidation and of Stereochemistry. Chemosphere 2000, 40, 767-773. [CrossRef]

3. Mahnik, S.N.; Rizovski, B.; Fuerhacker, M.; Mader, R.M. Determination of 5-Fluorouracil in Hospital Effluents. Anal. Bioanal. Chem. 2004, 380, 31-35. [CrossRef] [PubMed]

4. Mahnik, S.N.; Rizovski, B.; Fuerhacker, M.; Mader, R.M. Development of an Analytical Method for the Determination of Anthracyclines in Hospital Effluents. Chemosphere 2006, 65, 1419-1425. [CrossRef]

5. Tauxe-Wuersch, A.; De Alencastro, L.F.; Grandjean, D.; Tarradellas, J. Trace Determination of Tamoxifen and 5-Fluorouracil in Hospital and Urban Wastewaters. Int. J. Environ. Anal. Chem. 2006, 86, 473-485. [CrossRef]

6. $\quad$ Lenz, K.; Mahnik, S.N.; Weissenbacher, N.; Mader, R.M.; Krenn, P.; Hann, S.; Koellensperger, G.; Uhl, M.; Knasmüller, S.; Ferk, F.; et al. Monitoring, Removal and Risk Assessment of Cytostatic Drugs in Hospital Wastewater. Water Sci. Technol. 2007, 56, 141-149. [CrossRef]

7. Steger-Hartmann, T.; Kümmerer, K.; Schecker, J. Trace Analysis of the Antineoplastics Ifosfamide and Cyclophosphamide in Sewage Water by Twostep Solid-Phase Extraction and Gas Chromatography-Mass Spectrometry. J. Chromatogr. A 1996, 726, 179-184. [CrossRef]

8. Falter, R.; Wilken, R.-D. Determination of Carboplatinum and Cisplatinum by Interfacing HPLC with ICP-MS Using Ultrasonic Nebulisation. Sci. Total Environ. 1999, 225, 167-176. [CrossRef]

9. Buerge, I.J.; Buser, H.-R.; Poiger, T.; Müller, M.D. Occurrence and Fate of the Cytostatic Drugs Cyclophosphamide and Ifosfamide in Wastewater and Surface Waters. Environ. Sci. Technol. 2006, 40, 7242-7250. [CrossRef]

10. Llewellyn, N.; Lloyd, P.; Jürgens, M.D.; Johnson, A.C. Determination of Cyclophosphamide and Ifosfamide in Sewage Effluent by Stable Isotope-Dilution Liquid Chromatography-Tandem Mass Spectrometry. J. Chromatogr. A 2011, 1218, 8519-8528. [CrossRef]

11. Besse, J.-P.; Latour, J.-F.; Garric, J. Anticancer Drugs in Surface Waters: What Can We Say about the Occurrence and Environmental Significance of Cytotoxic, Cytostatic and Endocrine Therapy Drugs? Environ. Int. 2012, 39, 73-86. [CrossRef] [PubMed]

12. Jureczko, M.; Kalka, J. Cytostatic Pharmaceuticals as Water Contaminants. Eur. J. Pharmacol. 2019, 866, 172816. [CrossRef] [PubMed]

13. Johnson, A.C.; Jürgens, M.D.; Williams, R.J.; Kümmerer, K.; Kortenkamp, A.; Sumpter, J.P. Do Cytotoxic Chemotherapy Drugs Discharged into Rivers Pose a Risk to the Environment and Human Health? An Overview and UK Case Study. J. Hydrol. 2008, 348, 167-175. [CrossRef]

14. Xie, H. Occurrence, Ecotoxicology, and Treatment of Anticancer Agents as Water Contaminants. J. Environ. Anal. Toxicol. 2012. [CrossRef]

15. Gómez-Canela, C.; Ventura, F.; Caixach, J.; Lacorte, S. Occurrence of Cytostatic Compounds in Hospital Effluents and Wastewaters, Determined by Liquid Chromatography Coupled to High-Resolution Mass Spectrometry. Anal. Bioanal. Chem. 2014, 406, 3801-3814. [CrossRef]

16. Yin, J.; Yang, Y.; Li, K.; Zhang, J.; Shao, B. Analysis of Anticancer Drugs in Sewage Water By Selective SPE and UPLC-ESI-MS-MS. J. Chromatogr. Sci. 2010, 48, 781-789. [CrossRef]

17. Martín, J.; Camacho-Muñoz, D.; Santos, J.L.; Aparicio, I.; Alonso, E. Simultaneous Determination of a Selected Group of Cytostatic Drugs in Water Using High-Performance Liquid Chromatography-Triple-Quadrupole Mass Spectrometry. J. Sep. Sci. 2011, 34, 3166-3177. [CrossRef]

18. Negreira, N.; López de Alda, M.; Barceló, D. On-Line Solid Phase Extraction-Liquid Chromatography-Tandem Mass Spectrometry for the Determination of 17 Cytostatics and Metabolites in Waste, Surface and Ground Water Samples. J. Chromatogr. A 2013, 1280, 64-74. [CrossRef] 
19. Rabii, F.W.; Segura, P.A.; Fayad, P.B.; Sauvé, S. Determination of Six Chemotherapeutic Agents in Municipal Wastewater Using Online Solid-Phase Extraction Coupled to Liquid Chromatography-Tandem Mass Spectrometry. Sci. Total Environ. 2014, 487, 792-800. [CrossRef]

20. Franquet-Griell, H.; Cornadó, D.; Caixach, J.; Ventura, F.; Lacorte, S. Determination of Cytostatic Drugs in Besòs River (NE Spain) and Comparison with Predicted Environmental Concentrations. Environ. Sci. Pollut. Res. 2017, 24, 6492-6503. [CrossRef]

21. Gomez-Canela, C.; Cortes-Francisco, N.; Oliva, X.; Pujol, C.; Ventura, F.; Lacorte, S.; Caixach, J. Occurrence of Cyclophosphamide and Epirubicin in Wastewaters by Direct Injection Analysis-Liquid Chromatography-High-Resolution Mass Spectrometry. Environ. Sci. Pollut. Res. Int. 2012, 19, 3210-3218. [CrossRef] [PubMed]

22. Sottani, C.; Turci, R.; Micoli, G.; Fiorentino, M.L.; Minoia, C. Rapid and Sensitive Determination of Paclitaxel (Taxol $\left.{ }^{\circledR}\right)$ in Environmental Samples by High-Performance Liquid Chromatography Tandem Mass Spectrometry. Rapid Commun. Mass Spectrom. 2000, 14, 930-935. [CrossRef]

23. Gouveia, T.I.A.; Silva, A.M.T.; Ribeiro, A.R.; Alves, A.; Santos, M.S.F. Liquid-Liquid Extraction as a Simple Tool to Quickly Quantify Fourteen Cytostatics in Urban Wastewaters and Access Their Impact in Aquatic Biota. Sci. Total Environ. 2020, 740, 139995. [CrossRef]

24. Souza, D.M.; Reichert, J.F.; Martins, A.F. A Simultaneous Determination of Anti-Cancer Drugs in Hospital Effluent by DLLME HPLC-FLD, Together with a Risk Assessment. Chemosphere 2018, 201, 178-188. [CrossRef] [PubMed]

25. Kabir, A.; Furton, K.G. Fabric Phase Sorptive Extractors (Fpse). Available online: https://patents.google.com/patent/US2014027 4660A1/en (accessed on 15 May 2021).

26. Kumar, R.; Gaurav, H.; Malik, A.K.; Kabir, A.; Furton, K.G. Efficient Analysis of Selected Estrogens Using Fabric Phase Sorptive Extraction and High Performance Liquid Chromatography-Fluorescence Detection. J. Chromatogr. A 2014, 1359, 16-25. [CrossRef]

27. Kabir, A.; Mesa, R.; Jurmain, J.; Furton, K.G. Fabric Phase Sorptive Extraction Explained. Separations 2017, 4, 21. [CrossRef]

28. Tartaglia, A.; Kabir, A.; D’Ambrosio, F.; Ramundo, P.; Ulusoy, S.; Ulusoy, H.I.; Merone, G.M.; Savini, F.; D’Ovidio, C.; Grazia, U.D.; et al. Fast Off-Line FPSE-HPLC-PDA Determination of Six NSAIDs in Saliva Samples. J. Chromatogr. B 2020, $1144,122082$. [CrossRef]

29. Zilfidou, E.; Kabir, A.; Furton, K.G.; Samanidou, V. An Improved Fabric Phase Sorptive Extraction Method for the Determination of Five Selected Antidepressant Drug Residues in Human Blood Serum Prior to High Performance Liquid Chromatography with Diode Array Detection. J. Chromatogr. B 2019, 1125, 121720. [CrossRef] 